برآورد قدرت تركيب بِيرى عمومى و خصوصى صفات مورفولوزيك و عملكرد دانه در كَندم نان

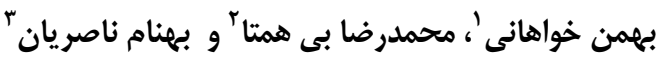

ا- دانشآموخته كارشناسى ارشد، كروه زراعت و اصلاح نباتات دانشخاه تهران و دانشجوى دكترى، بخش زراعت و واصلات الاح نباتات دانشكاه شيراز

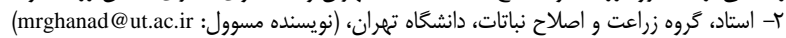

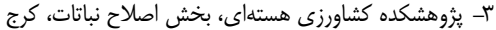

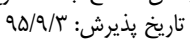

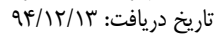

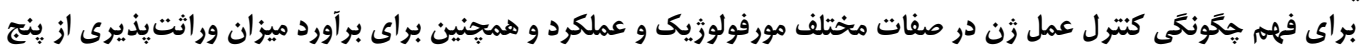

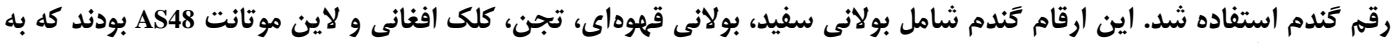

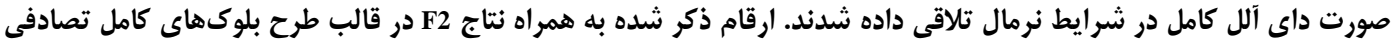

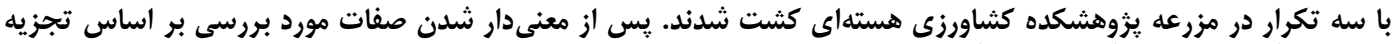

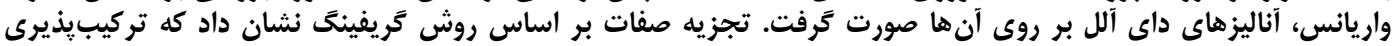

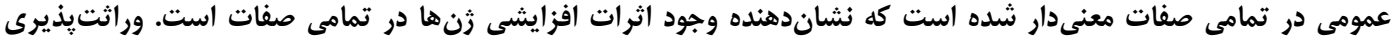

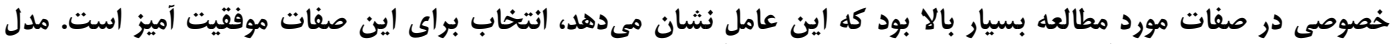

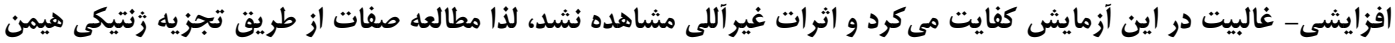

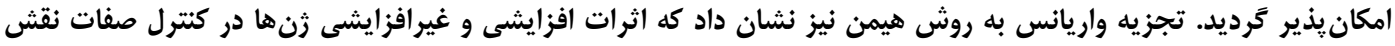

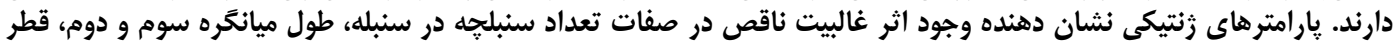

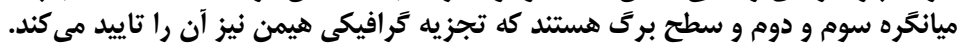

وازههاى كليدى: اثر افزايشى، تجزيه داى آلل، كريفينَّ، تَندم، هيمن هيمن نيز ان

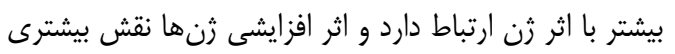

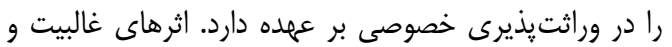

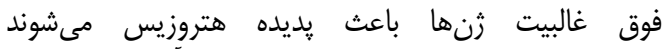

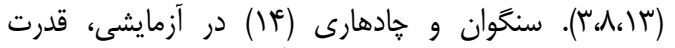

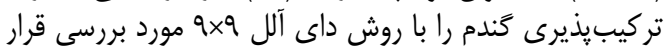

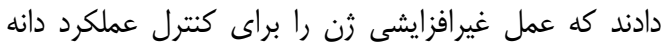

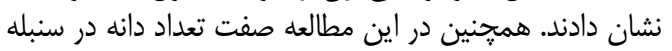

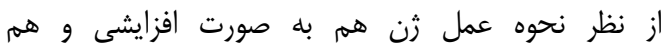

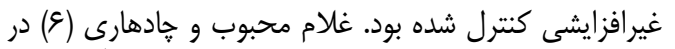

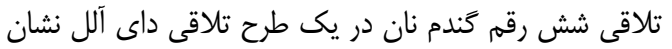

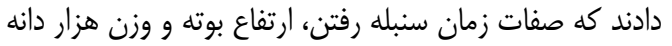

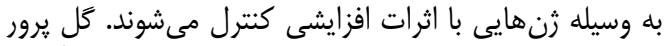

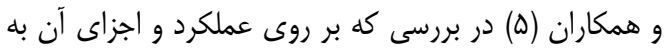

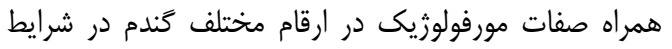

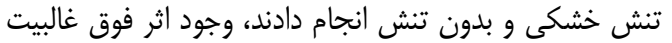

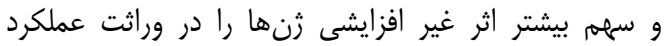

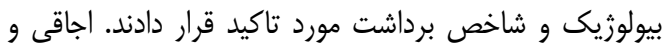

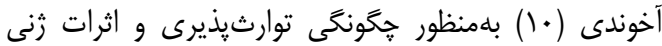

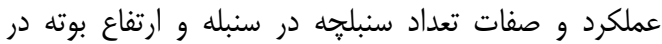

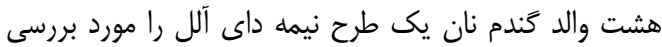

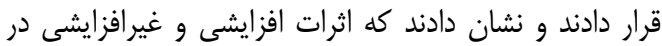

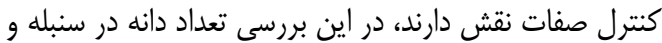

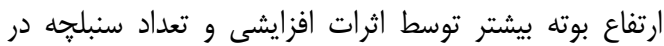

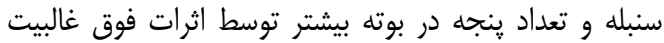

$$
\text { زنها كنترل مىشوند. }
$$

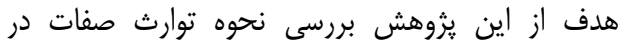

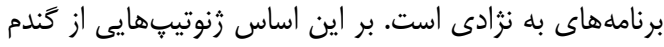

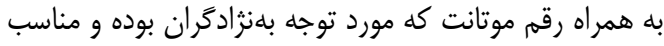

\section{كَّندم دومين غله در دنيا بعد از برنج است، كه محصولات}

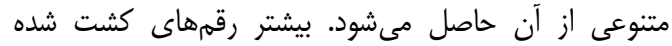

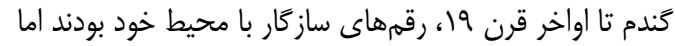

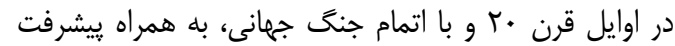

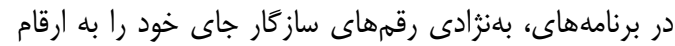

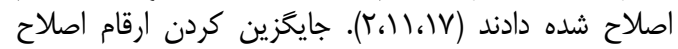

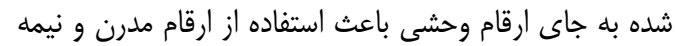

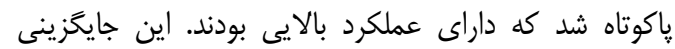

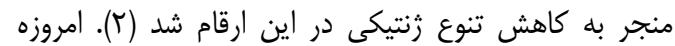

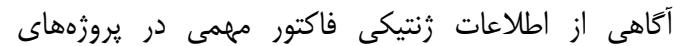

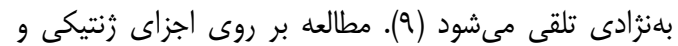

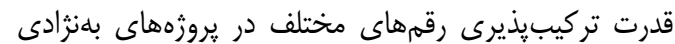

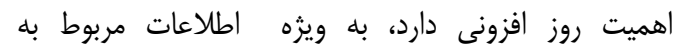

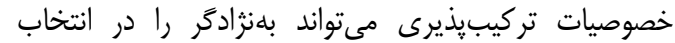

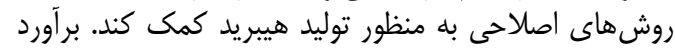

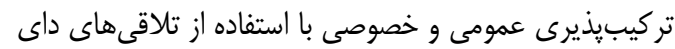

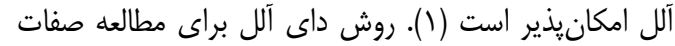

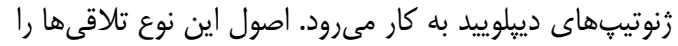

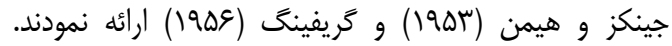

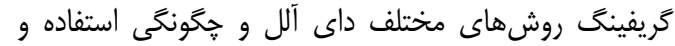

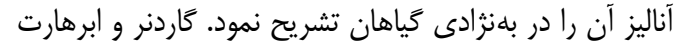

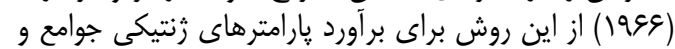

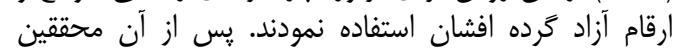

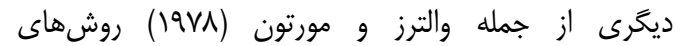
ييشرفتهترى براى آناليز داى آلّل ارائه نمودند. روش دائ دآلآلل

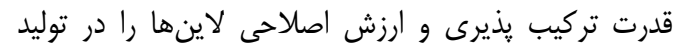

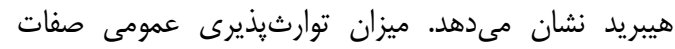


خط ركرسيون و بر اساس نرمافزار

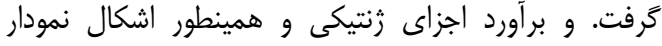
كرافيكى هيمن بر اساس نرماف افزار

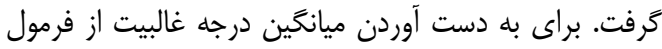
رو به رو استفاده شد. كه در فرمول بالا H1 واريانس ناشى از اثر غالبيت براى إئائ

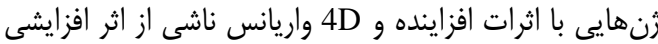

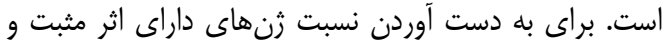
م2 $\overline{4 \mathrm{H} 1}$

در فرمول فوق H2 هم واريانس ناشى از اثر غالبيت براى

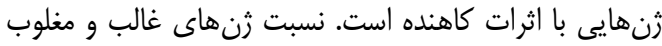
در والدين از فرمول زير بدست آمد. $1 / 2 \sqrt{4 \mathrm{DH} 1-1 / 2 \mathrm{~F}}$

در فرمول فوق F ميانكين كواريانس اثرات افزايشى و غالبيت

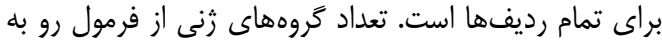
رو به دست أمد. $\overline{\mathrm{H} 2}$

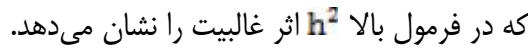
وراثتيذيرى خصوصى از فرمول زير محاسبه شد.

$\mathrm{h}_{\mathrm{n}}^{2}-\frac{1 / 4 \mathrm{D}}{\frac{1}{-1} \mathrm{D}+\frac{1}{16} \mathrm{H} 1-\frac{1}{\mathrm{~g}} \mathrm{~F}+\mathrm{E}}$

در اين فرمول h²n، وراثتيذيرى خصوصى و E واريانس محيطى است (V) (V).

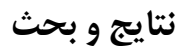

تجزيه واريانس براى صفات مورد بررسى انجام و در

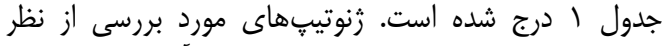

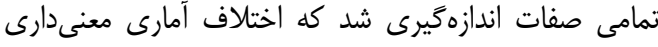

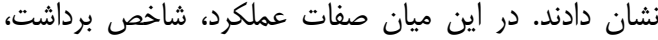
طول ميانكره دوم و سوم، قطر ميانكره دوم و و سوم، سطح دانح

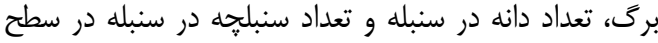

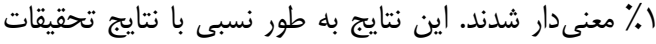

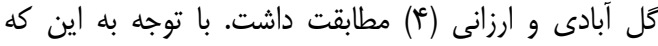

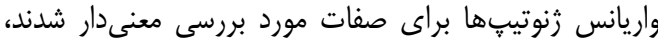

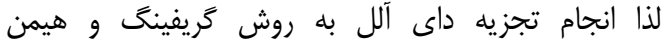

$$
\text { امكانيذير است. }
$$

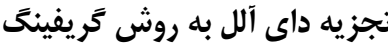

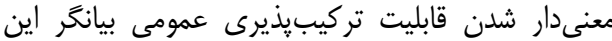

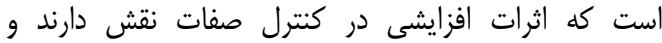

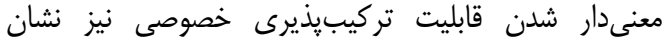

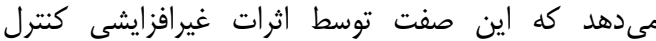

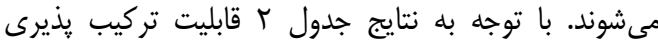

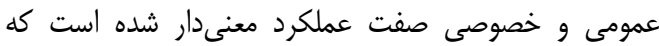

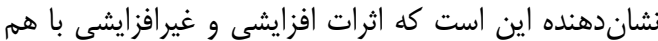

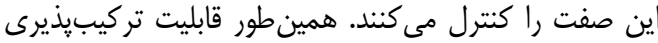
عمومى صفات شاخص برداشت، طول ميانكره دوم و سوم، تركي
منطقه جنوب شرق ايران مىباشند، براى تجزيه زنتنيكى

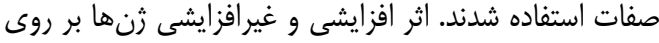
صفات مورد بررسى قرار كرفت.

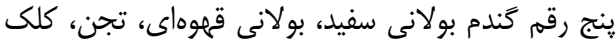

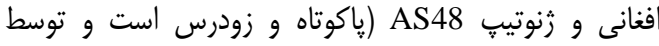

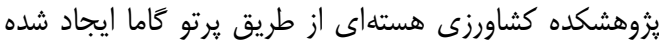

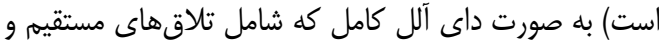

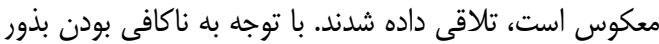

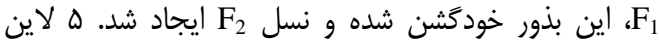

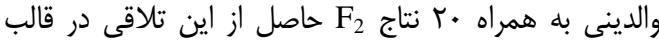

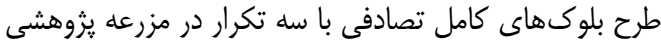

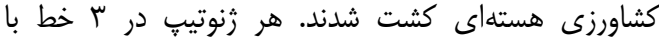

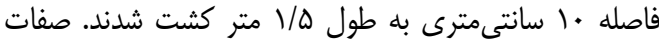

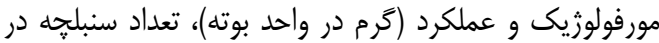

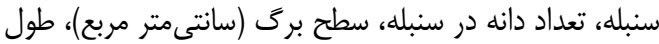

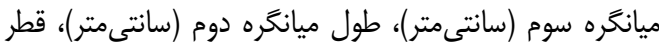

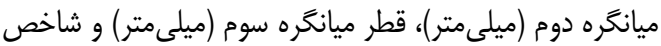

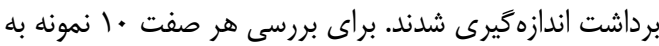

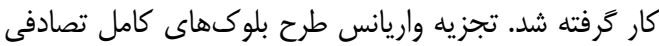

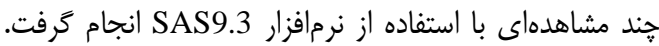

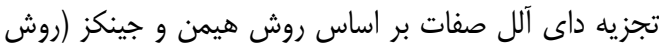

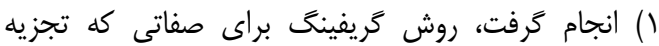

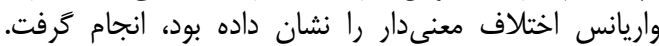

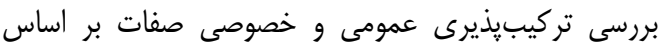

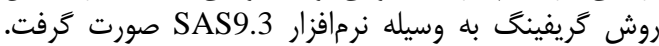

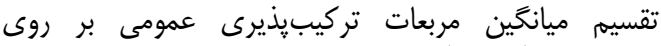

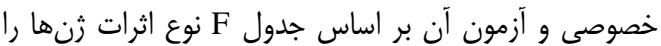
مشخص مى كند كه نوع عمل زن باس با فرمول زير به دست آمدات آندا

\section{$M S(G C A)$}

\section{$M S(S C A)$}

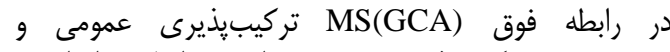
MS(SCA) و واريانس غالبيت از طريق فرمول زير محاسبه كرديديد. $\sigma_{\mathrm{G} C \mathrm{~A}}^{2}=\left(\frac{1+\mathrm{F}}{4}\right) \sigma_{\AA}^{2}$

$\pi_{\mathrm{E}: \mathrm{A}}^{2}=\left(\frac{1+\mathrm{F}}{2}\right) \pi_{\mathrm{D}}^{2}$

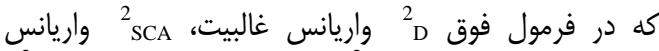
تركيبذيذيرى خصوصى،

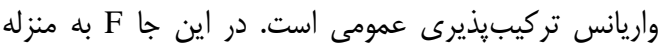

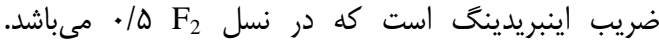
وراثتيذيرى خصوصى با استفاده از فرمول زير محاسبه $\mathrm{h}^{2}=\frac{\sigma_{\mathrm{A}}^{2}}{\sigma_{\mathrm{p}}^{2}}$

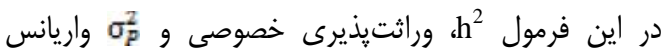
فنوتييى است. تجزيه صفات بر اساس روش هيمن و شيب 
است و ميزان وراثتيذيرى خصوصى در اين صفات كم است.

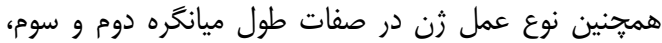

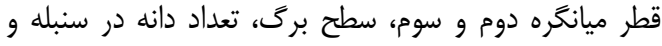

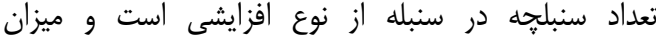

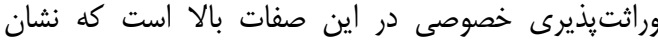

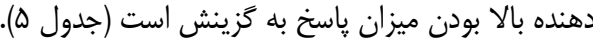

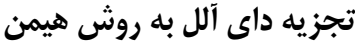
براى انجام تجزيه داى آلى آل به روش رئ هيمن اول بائ بايد

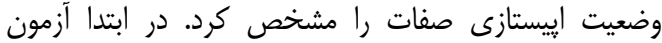

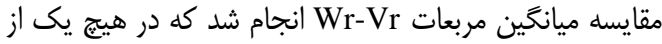

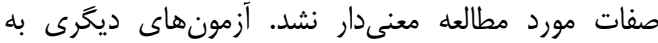

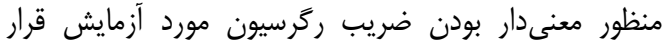

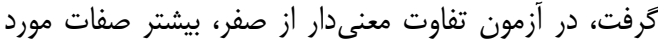

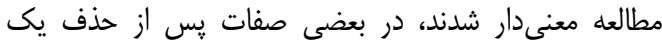

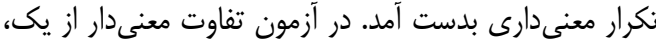

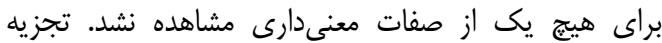

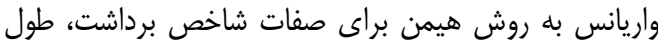

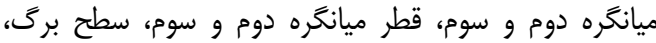

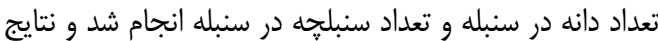

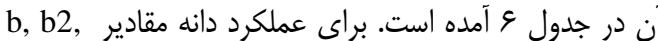

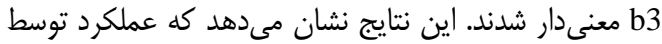

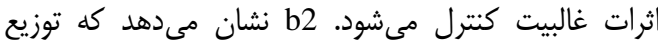

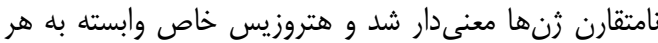

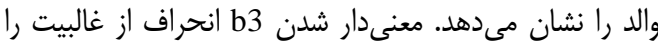

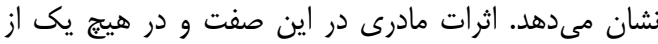

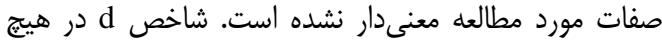

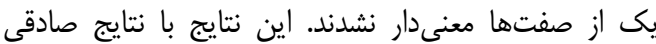

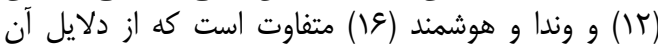

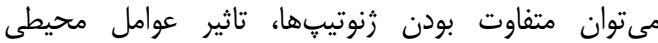
همجون شرايط كشت و شرايط اقليمى متفاوت و و اشتباهات

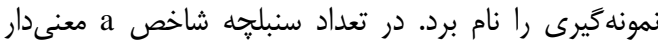

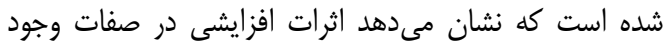
دارد و با نتايج صادقى (ז) مان متفاوت است.
قطر ميانكره دوم و سوم، سطح برك، تعداد دانه در سنبله و

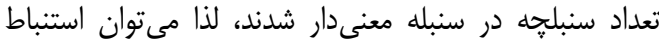

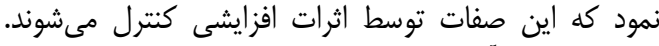

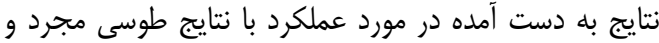

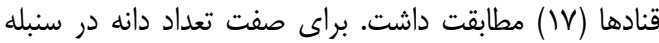

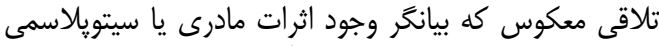

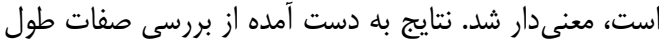

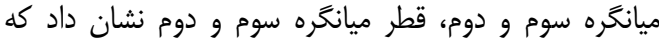

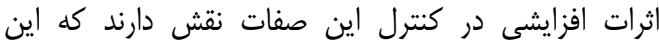

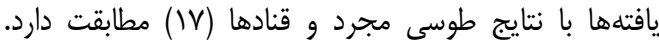

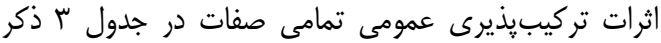

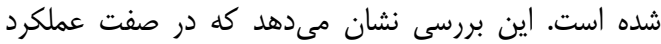

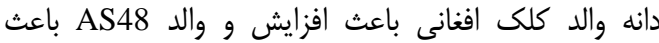

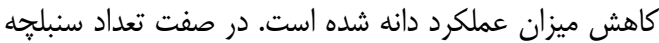

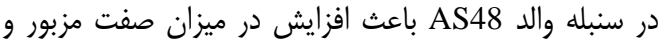

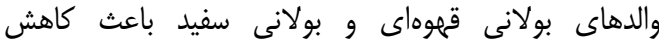
معنى دارى در تعداد سنبلجه در سنبله

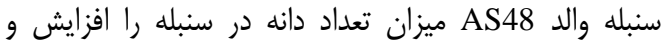

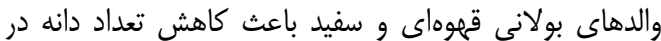

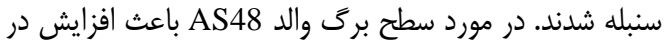

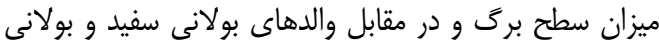
قهوماى باعث كاهش سطح برى

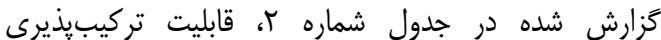

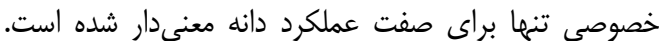

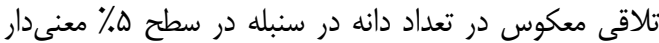

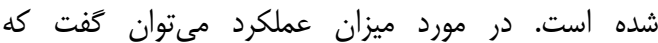
تلاقى هاى بولانى قهوماى × AS48 و بولانى سفيد × AS48

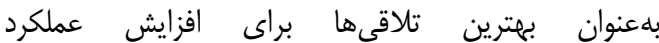

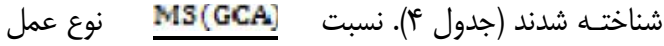

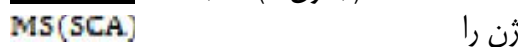
مشخص مى كند، بيشتر بودن اين نسبت نشاندهنده نقش

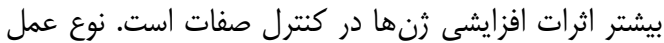

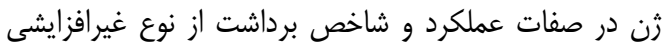

Table 1. Analysis of Variance of Morphological and Yeld Traits

جدول (- تجزيه واريانس صفات مورفولوزيك و عملكرد گندم نان

\begin{tabular}{|c|c|c|c|c|c|c|c|c|c|c|}
\hline شرداشت & طول ميانكره & طول ميانكره & قطر ميانكره & قطر ميانكره & سرى & تعداد دانه در & سنبلجه & عملكرد & آزادى & تنيير \\
\hline $\mid \Psi / \Delta / /^{* * * *}$ & $101 / 90^{* * * 3}$ & $\mid \Lambda \Delta / V \Lambda^{*}$ & $T / 1 Q$ & $r / r^{* * * *}$ & $|r V \Delta / \Delta|^{* * * 3}$ & Trq/Ve & $|r / 4|$ & $\Delta I V / A T^{*}$ & $r$ & تكرار \\
\hline TEY $/\left.\cdot\right|^{* * * *}$ & $|M N / r|^{\text {*⿻ }}$ & 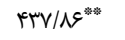 & $r / \Lambda r^{* * *}$ & $1 /\left.\Delta\right|^{* * n}$ & 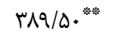 & IVDT/AV" & $r M / r r^{* 0 *}$ & $1 \cdot \Delta / \mu^{\prime}$ & re & تيمار \\
\hline $11 \% / 9$ & rT/rq & $\lceil N / F q$ &.$/ \$ \Delta$ & ع & १९/Dร & $r \cdot N / \Lambda \Gamma$ & $r / \Delta S$ & $10 \cdot / 1 T$ & $1 \wedge$ & خطا \\
\hline$\%$ q & سז\% & $\%$ r. & $\% 10$ & سו & سז\% & \% & $\%$. & سז\% & & ضغريبرات \\
\hline r/sq & $1 \% / 9$ & $r r / Q \Lambda$ & $F / \leftarrow \wedge$ & $\varphi / \Delta \Delta$ & $r q / 1 q$ & $9 \Delta / M$ & YI/RG & $r \vee / 1$ & & ميانكين \\
\hline
\end{tabular}




\begin{tabular}{|c|c|c|c|c|c|c|c|c|c|c|}
\hline شراشت & طول ميانگره & طول ميانگره & ميانكره & ميانكره & $\begin{array}{l}\text { سطح برى } 1 \text { سانتىمتر } \\
\text { مربع) }\end{array}$ & تعداد دانه در & سنبلهاد & در واحد بوته) (كرم) & آزادى درجه & منابع تغيير \\
\hline$\Delta Q / \Delta Q^{*}$ & $\mid r g / F V^{*}$ & $r r V / V I^{* * *}$ & $\cdot|\Lambda|^{* * *}$ & . & 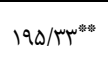 & $948 / 1 .{ }^{* * *}$ & $\mid r / 9 D^{* *}$ & $r Q T / r \Lambda^{*}$ & r & عموميىئى \\
\hline$\Delta \Delta / \Gamma q$ & F/AD & $\mathrm{V} / \mathrm{lf}$ & $.1 \cdot 0$ & $.1 \cdot 1$ & Tr/QT & $V G / T V$ &.$/ v 9$ & $|\mathrm{~V}| \cdot \mathrm{N}^{\text {*a*n: }}$ & 1. & خصوصى خيذيرى \\
\hline$q / q r$ & $1 / \pi F$ & 1/9 &.$/ . r$ & .1 .4 & $r \pi / .1$ & $M / Q N^{*}$ & ./Va & ه & 1. & معكوسى تلاقى \\
\hline Tr/gN & T/F & $V / 1$. & $.1 \cdot 0$ & .1 .4 & $15 / 19$ & Tr &.$/ 94$ & $81 / v$. & is & خطا \\
\hline
\end{tabular}

نشاندهنده وجود تقارن بين زنهاى مثبت و منفى است. در

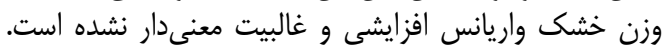

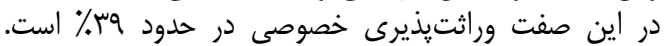

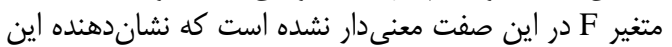

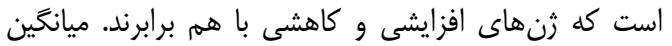

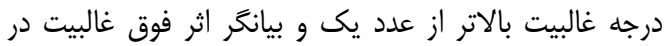

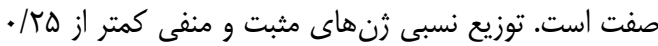

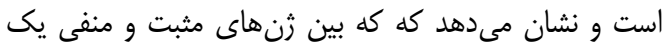

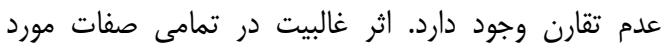

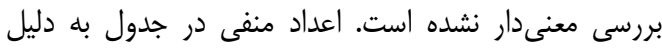

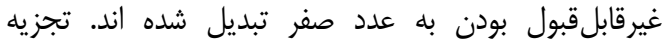

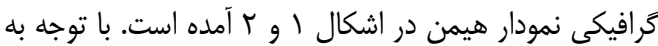

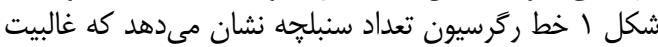

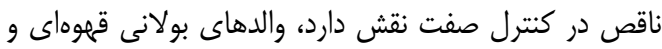

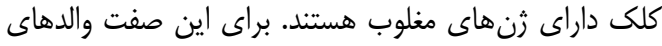

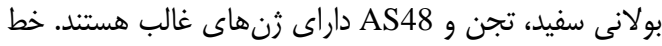

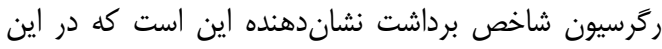

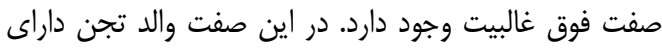

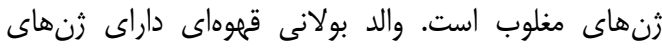
غالب و مغلوب است. والدهاى بولانى سفيد، كلى ونى و

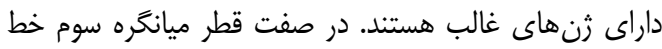

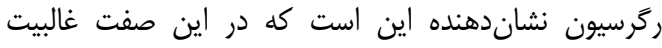

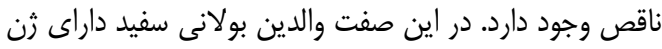

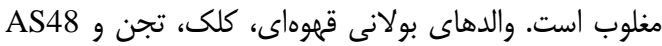

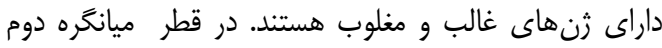

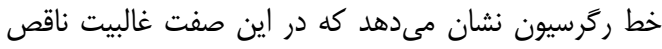

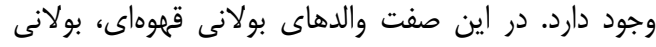

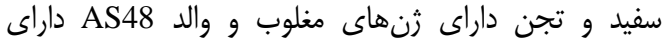

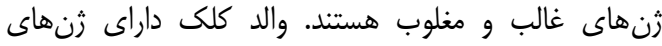

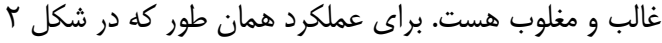

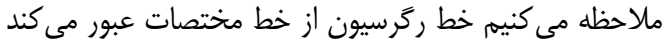

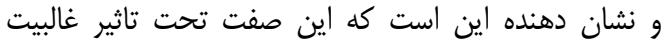

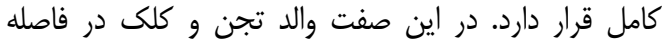

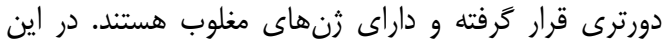

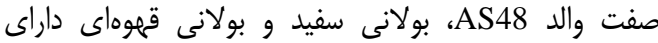

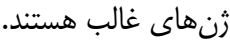

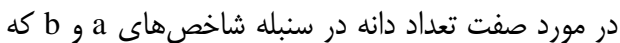
نشان دهنده اثرات افزايشى و غالبيت هستند، معنى دار شدند.

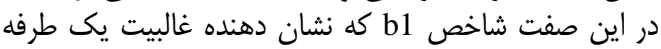

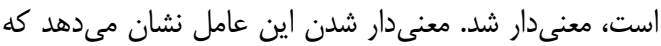

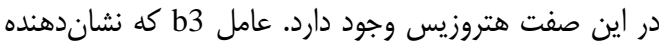

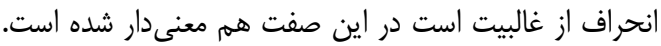

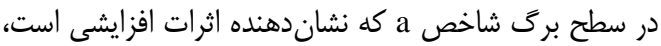

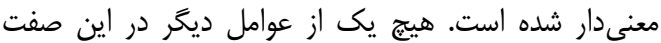

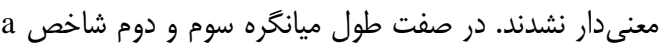

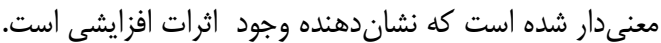

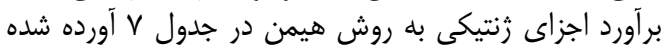

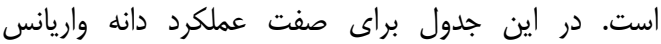

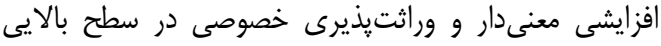

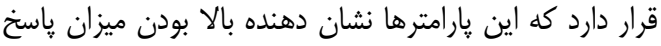

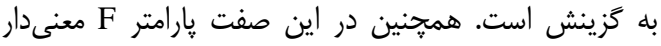

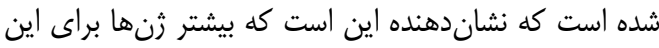

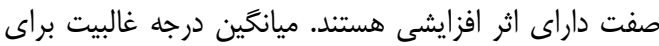

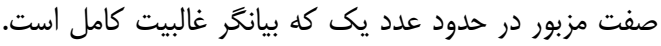

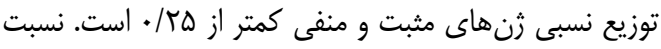

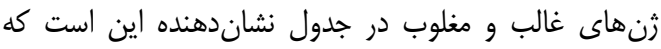

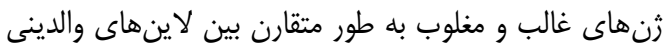

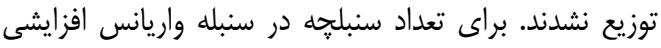

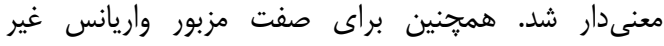

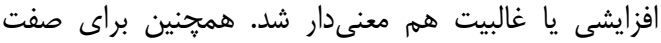

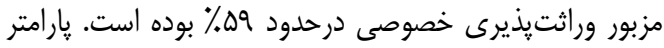

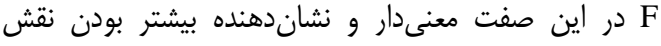

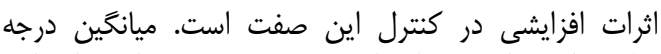

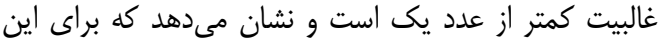

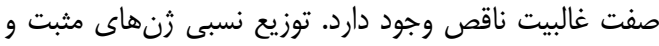

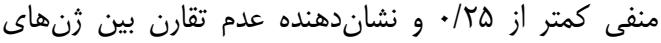

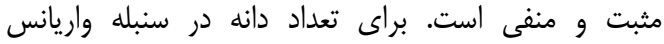

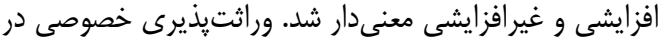

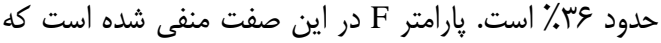

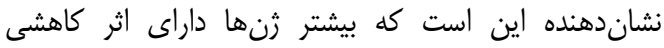

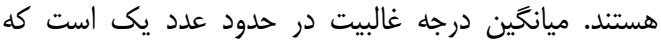

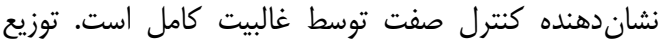
نسبى ثنهاى مثبت و منفى در حدود عدد هان/ • است و 
Table 3. GCA values for all traits

جدول ؟ـ- مقادير تر كيبيذيرى عمومى براى تمام صفات مورد بررسى

\begin{tabular}{|c|c|c|c|c|c|c|c|c|c|c|}
\hline شرداشت & $\begin{array}{c}\text { طول ميانكره } \\
\text { (سانتىمتر) }\end{array}$ & طول سوانتره & 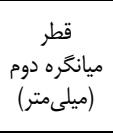 & 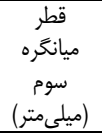 & 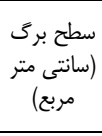 & تعر سنبله & سنبلجه & 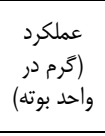 & تعداد ينجها & والدين \\
\hline$-1 / 4$ & $\Delta / \cdot r^{\omega *}$ & s/r"w. & $-q / \cdot \Gamma^{* * * *}$ & $-8 / 4 q^{6 / 3}$ & $-F / 1 q^{* * w}$ & $-\Gamma / \Gamma \Lambda^{* * *}$ & $-91.9^{* * * *}$ & $-1 / \mu$. & $r / \cdot r^{* \prime \prime}$ & قبولانى \\
\hline$-1 / 49$ & $r / W v^{* * *}$ & $r / \cdot r^{* * a}$ & $-r / q \Psi^{*}$ & $-F / \Delta \Lambda^{* *}$ & $-r / r \cdot *$ & $-4 / . .^{* *}$ & $-r / \cdot \Gamma^{*}$ & $-1 / T r$ &.$/ 91$ & سبفيدى \\
\hline$r / \Lambda \cdot * * *$ & $-\Gamma / \Gamma^{* * *}$ & $-1 / 9 T$ & 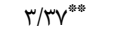 & $r / r^{s^{* a n}}$ & $-1 / V V$ & I/VE & .199 & $1 / 9 V$ &.$-|r|$ & تجن \\
\hline$\cdot / v \wedge$ & $r / V \Lambda^{* 0 .}$ & $f / r r^{* * a}$ & $r / r^{r}$ & $r / \Gamma D^{* \ldots}$ & $1 / 91$ & $-1 / 9 T$ & $-\cdot|r|$ & $r / \cdot \Lambda^{* * * a t}$ & 1/8. & كلك \\
\hline-.191 & $-9 / \Psi^{* * *}$ & $-1 \Gamma / 99^{* * *}$ & $1 / 9 V$ & $\Gamma / \Delta \Gamma^{* * *}$ & $8 / r^{* \text { wath }}$ & 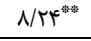 & 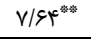 & $-r / \mu^{*}$ & $-F /\left.F\right|^{* * *}$ & AS48 \\
\hline
\end{tabular}

جدول عا- مقادير تركيب يذيرى خصوصى و تلاقى معكوس دورى ها براى صفات عملكرد و تعداد دانه در سنبله Table 4. SCA and REC values for Yeld and number of grains per spike

\begin{tabular}{|c|c|c|c|}
\hline تعداد دانه در سنبله & تلاقى معكوس دوركها & عملكرد (كَرم در واحد & دوركها و والدين \\
\hline.$- / 9 \Delta$ & بولانى سفيد×بولانى قهوهاى & $1 / \Delta 9$ & بولانى قهوهاى ×بولانى سفيد \\
\hline.$- / 99$ & تجن ×بولانى قهوهاى & $-1 / 49$ & بولانى قهوماى × تجن \\
\hline.$/ 1$ & كلك ×بولانى قهوهاى &.$- / \Delta S$ & بولانى قهوهاى×كلى \\
\hline $1 / 4 \wedge$ & 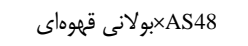 & $r / r T^{*}$ & بولانى قهوهاى ×AS48 \\
\hline.$- / 8 \mathrm{~V}$ & تجن×بولانى سفيد & $-\cdot / \Gamma \Delta$ & بولانى سفيدxتجن \\
\hline.$- .1 \cdot 1$ & كلك×بولانى سفيد & $-r / \Delta Q^{*}$ & بولانى سفيد×كلى \\
\hline.$/ 9 V$ & PبS48×بانى سفيد & $\gamma / \cdot \Delta *$ & بولانى سفيد×AS48 \\
\hline$-r / T V^{*}$ & كلك×تجن & $1 / .9$ & تجن تكلى \\
\hline$-1 / r$ & AS48×AS48 & $-r / 9 e^{* \prime \prime}$ & تجن تج48S \\
\hline$r / \Lambda \varepsilon^{* *}$ & 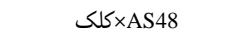 &.$|9|$ & كلى×AS48 \\
\hline
\end{tabular}

جدول هـ- نسبت ميانخين مربعات GCA به SCA و همجنين نوع عمل زن، واريانس افزايشى، واريانس غالبيت و وراثتيذيرى خصوصى Table 5. The mean square ratio of GCA to SCA as well as gene action, additive variance, dominance variance and heritability

\begin{tabular}{|c|c|c|c|c|c|}
\hline وراثتيذيرى خصوص & واريانس غالبيت & واريانس افزايشى & نوع عمل خن & $\begin{array}{l}M S(G C A) \\
M S(S C A)\end{array}$ & صفات \\
\hline.$/ I V$ & FN/AT & $T H / H^{2}$ & غير افزايشى & $T / 4 V$ & عملكرد \\
\hline.$|\lambda|$ & .1 .98 & $r / \backslash 9$ & افزايشي & $\mid 8 / \cdot 1^{\text {*wtin }}$ & تعداد سنبلحه \\
\hline$\cdot / v q$ & $1 F / \Lambda \Delta$ & $r$ rq/VF & افزايشى & $\mid r / T V^{* i k i n}$ & تعداد دانه در سنبله \\
\hline.$/ v r$ & $r / v r$ & $F / 1 \Lambda$ & افزايشى & N/98**: & سطح برى \\
\hline .1 .4 & $\mid F / \Delta F$ & $1 / \Delta V$ & غير افزايشى & $1 / \cdot V$ & شاخص برداشت \\
\hline.$/ 94$ & $.1 \cdot 10$ & $M / \backslash S$ & افزايشى & $F V / \tau^{* 0 *}$ & طول ميانكَره سوم \\
\hline$\cdot / M$ & - /AVQ & $\Gamma \Delta / 1$. & افزايشى & $r N / T^{m}$ & طول ميانكَره دوم \\
\hline$\cdot / \Lambda$. &.$/ 10$ & . & افزايشى & $11 / g^{*}$ & قطر ميانكره سوم \\
\hline$\cdot / 19$ & $\cdot / . \cdot 1 f$ & $\cdot / r$. & افزايشى & 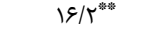 & قطر ميانگره دوم \\
\hline
\end{tabular}


جدول و- تجزيه واريانس صفات مورد نظر بر اساس تلاقى داى آلل هيمن

\begin{tabular}{|c|c|c|c|c|c|c|c|c|c|c|}
\hline شاخص برداشت & $\begin{array}{c}\text { طول ميانكتره دونتر) } \\
\text { (سانتى }\end{array}$ & 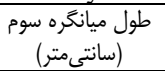 & 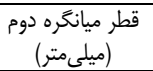 & 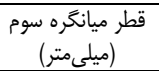 & 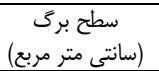 & تعداد دانه در سنبله & تعداد سنبلهِ & عملكرد (كرم در واحد) & آَازدى & منابع تغييرات \\
\hline$\Delta Q / \Delta Q$ & $\mid r g / 4 v^{*}$ & $r T V / V I^{*}$ & 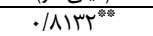 & $. / 94 \wedge)^{* * 3}$ & $19 \Delta / \Delta \gamma^{*}$ & $998 / 1$. & $|r / q|^{*}$ & TOT/TA & f & $\mathrm{a}$ \\
\hline$\Delta \Delta / \varphi^{* \prime \prime}$ & $r / \Lambda \Delta$ & V/If & $.1 . \Delta 9 V$ &.$/ .119$ & $T T / \Delta S$ & $V G / V^{*}$ & . /vares & $|V| / \cdot \wedge^{*}$ & 1. & b \\
\hline$r \Delta / r)$ & TV/IS & $+1 / \div 1$ &.$/ \cdot 19$ & ה א.r/ & $r T / \mathcal{T V}$ & $19 T / \pi T^{*}$ & . &.$/ 4 \lambda$ & 1 & b1 \\
\hline$\Delta s / F s^{\prime \prime}$ & T/VG & Q/9F & . & ع عץ+/. & Tr/TI & 1.119 & $.|\Delta| \Delta \mid$ & $19 \Lambda / \Delta 9^{*}$ & f & b2 \\
\hline$\Delta N / r \cdot *$ & $r / \cdot V$ & $1 / T r$ & $\cdot / 1 \cdot r \Delta^{* * *}$ & $.1 .94)^{*}$ & $I T / N$ & $1 \cdot \Delta / 9 r^{*}$ & $1 / \cdot 0 \cdot 1^{*}$ & $\mid \Lambda \mu / r \cdot *$ & $\Delta$ & b3 \\
\hline D/R & r r r r r & I/ve & $\cdot 1 \cdot r+1$ &.$/ . v q$ & IV/Ar & $1 .+/ r$. & 年 & SQ/DT & f & c \\
\hline س./ &.$/ 99.4$ & $r / \cdot \kappa^{c}$ & תسזץ./. & $.1 .9 \mathrm{VA}$ & ז//94 & $V N / A F$ & צr../1 & $q \Psi / \Delta V$ & 8 & d \\
\hline
\end{tabular}

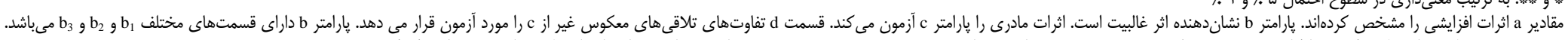

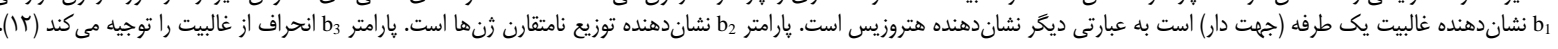

Table 7. Estimated the Genetic Component in Hayman method

جدول V- برآورد اجزاى زنتيكى در روش هيمن

\begin{tabular}{|c|c|c|c|c|c|c|c|c|c|}
\hline قطر ميانكره & قطر ميانكره & طول ميانكره & طول ميانكره & 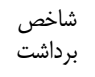 & 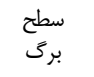 & تعداد دانه در & سنبله & 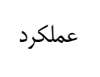 & دورَها و والدين \\
\hline$\cdot / 11^{\prime \prime}$ & $\cdot / 1 r^{*}$ & $\mid \varepsilon / \Lambda T^{*}$ & $F \Delta / \cdot \Delta^{*}$ & $r \varepsilon / \Delta q^{*}$ & $r N / r^{*}$ & $1 \cdot T / \gamma^{*}$ & $1 / w V^{\prime \prime}$ & $|1| \varepsilon / \cdot Y^{*}$ & D(واريانس افزايشى) \\
\hline$\cdot 1 \cdot \Lambda^{\prime \prime}$ & $\cdot / \mu$ & $r \mid / \varphi^{*}$ & $\Delta Q / \sim V^{*}$ & $\mid r T / T \omega^{*}$ & $1 . F / \lambda F$ & $f \cdot 9 / \kappa r^{*}$ & $r / \pi r^{* \prime \prime}$ & $\Delta \omega \cdot / \xi$ & H1(واريانس غالبييت) \\
\hline - & - & - & • & sQ/VA & Irr/Qצ" & $\Delta 99 / 9)^{*}$ & $r / \Delta r^{*}$ & rav/A. & H2(واريانس غالبيت) \\
\hline$\cdot / \varphi^{\prime \prime}$ &.$/ 1 Q^{*}$ & $1 / 9 \Lambda^{*}$ & $\mathrm{rN}^{\prime} / \mathrm{V}^{\mathrm{*}}$ & $V \Delta / \wedge q^{*}$ & $1 r / 99$ & 吾 & $1 / r^{*}$ & $r \cdot \Delta / \kappa^{*} \Delta^{*}$ & F (اثر متقابل اثر افزايشى و غيرافزايشى) \\
\hline$\cdot / 41$ & $\cdot / V \Delta$ & $\cdot / \Delta F$ & $\cdot / \Delta V$ & $1 / \cdot v$ &.$/ 98$ &.$/ 99$ & $\cdot / v 8$ & $1 / \cdot 1$ & (ميانكَين درجه غالبيت) \\
\hline.$/ 91$ & $1 Q / T V$ & 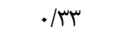 & 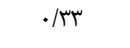 & - & . & • & • & - & |تعداد كروه هاى ثنى) \\
\hline . & . & . & . & rו/. & $\cdot / \pi \Delta$ & $\cdot / \pi \Delta$ & $\cdot|r|$ & .111 & 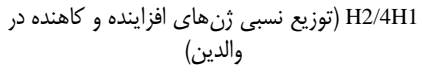 \\
\hline - & N/rq & 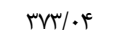 & $T V / F$. & · & $1 / 91$ & $\cdot / N T$ & $r / 90$ & · & نسبت زن هاى غالب و مغلوب در والدين \\
\hline .119 & .199 & ./9T & $1 / \cdot$ & . & שא/. & 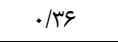 &.$/ 09$ & 年 & h2n(وراثت يذير خصوصى) \\
\hline
\end{tabular}


จ9

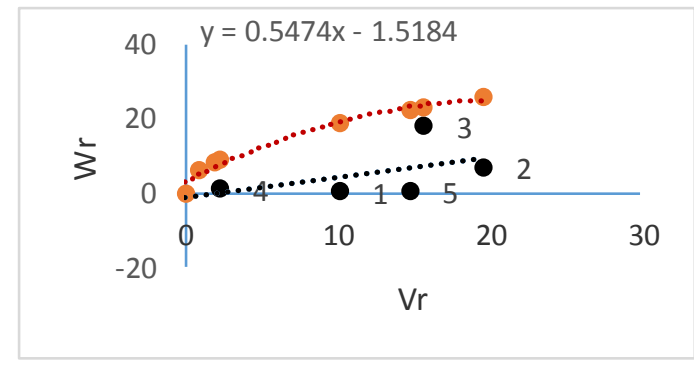

شاخص برداشت

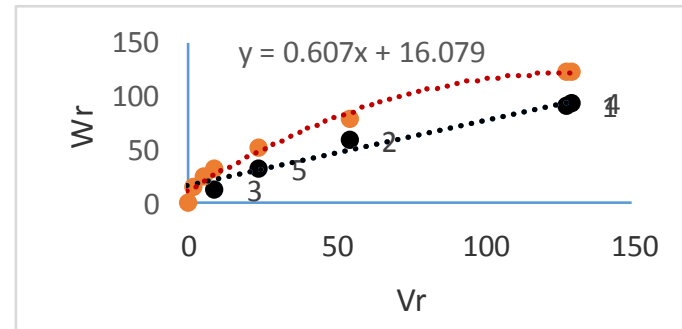

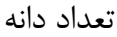

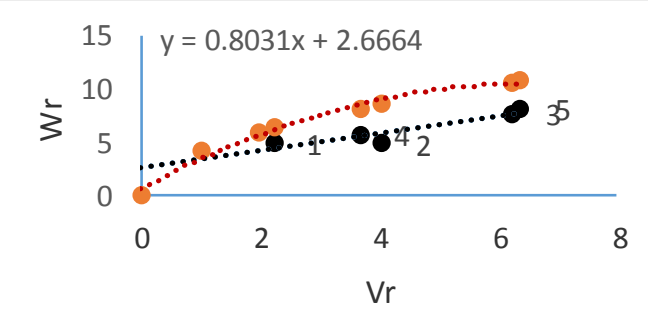

طول ميانخره دوم

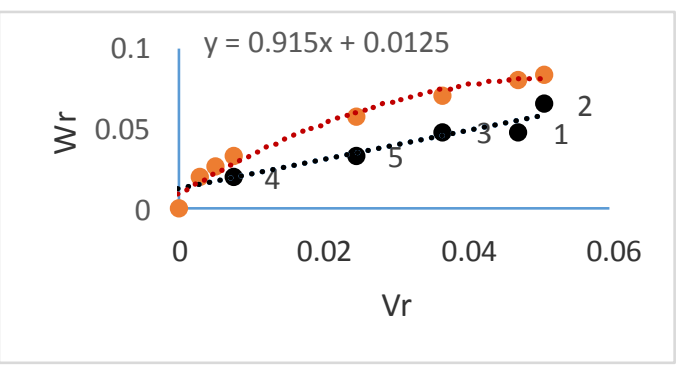

قطر ميانگره دوم
يثوهشنامه اصلاح گياهان زراعى/ سال دهم/ شماره هr/ بهار Vوسו .

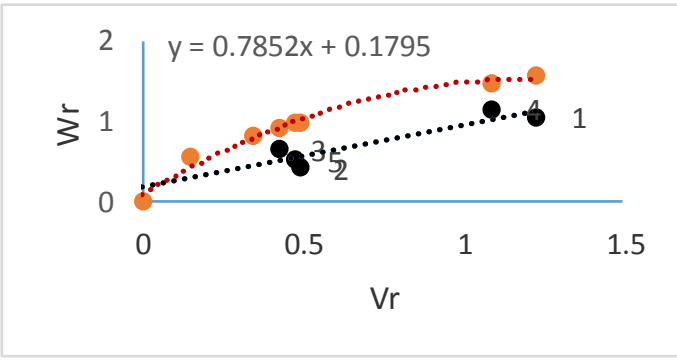

تعداد سنبلجه

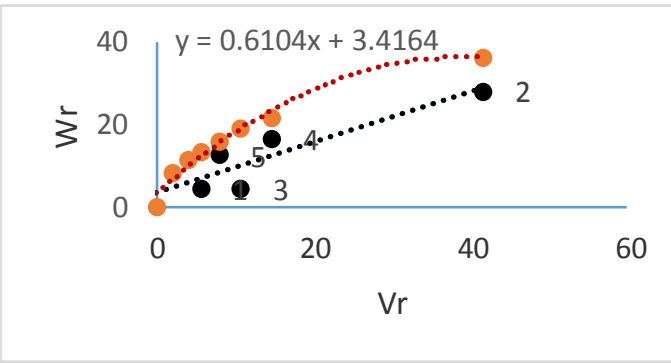

$$
\text { سطح برى }
$$

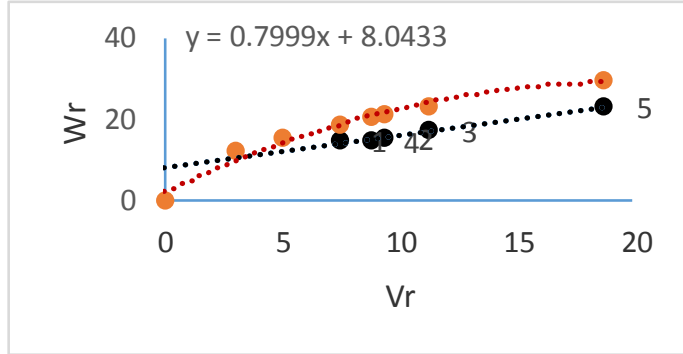

طول ميانگره سوم

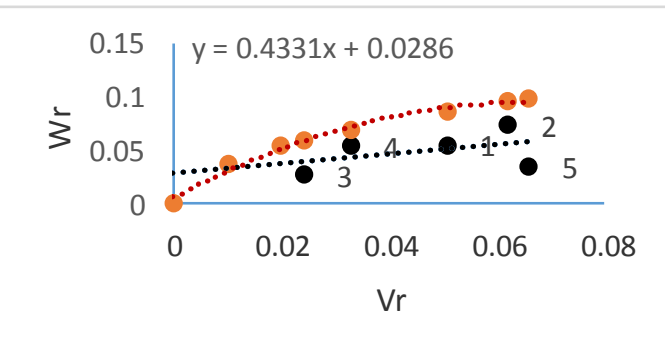

$$
\text { قطر ميانگره سوم }
$$

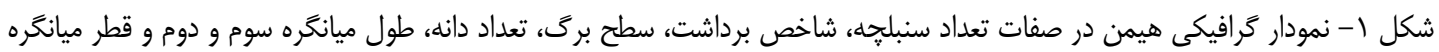

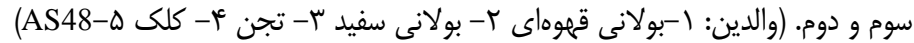

Figure 1. Hayman's graphical charts for number of spikelet, harvest index, leaf area, number of grains, second and third internodes length and second and third internodes diameter. (1- Brown Bolani, 2- White Bolani 3- tajan 4-Kealk 5- AS48) 


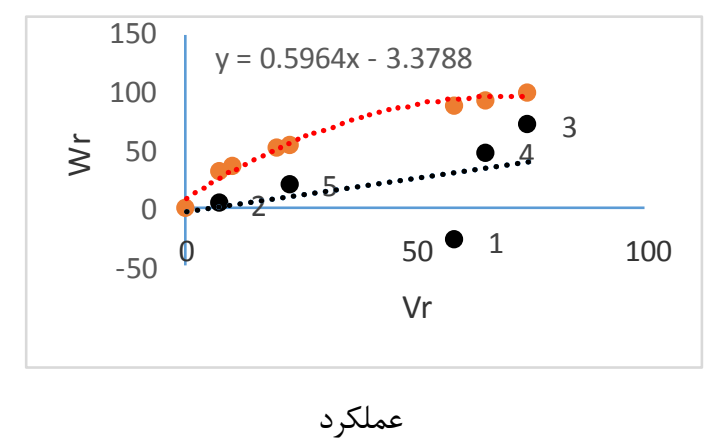

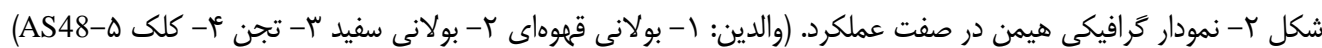

Figure 2. Hayman's graphical charts for grain yield. (1- Brown Bolani, 2- White Bolani 3- tajan 4-Kealk 5- AS48)

صورت توام در كنترل بيشتر صفات دخيل بودند و بيشتر صفات توسط غالبيت ناقص كنترل شدند.

تشكر و قدردانى

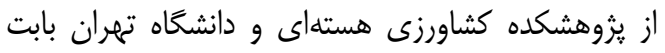

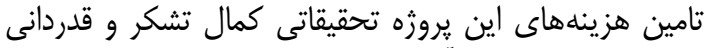

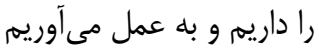

تجزيه داى آلل به روش كريفينگ براى تمامى صفات

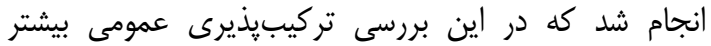

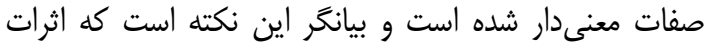

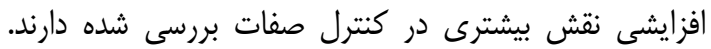

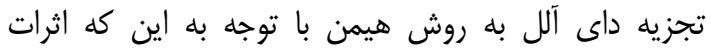

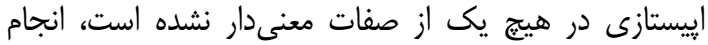
كرفت و بر اساس اين اناليز اثرات افزايشى و غالبيت بنده إنهان 
1. Ahmadi, J., A.A. Zali, B. Yazdi-samadi, A. Talaie, M.R. Ghannadha and A. Saeidi. 2003. A Study of Combining Ability and Gene Effect in Bread Wheat under Drought Stress Condition by Diallel Method. Iranian Journal Agriculture Science, 34:1-8 (In Persian).

2. Bordes, J., G. Branlard, F.X. Oury, G. Charmet and F. Balfpurier. 2008. Agronomic characteristics, grain quality and flour rheology of 372 bread wheats in a worldwide core collection. Journal of Cereal Science, 48: 569-579.

3. Derikvand, R., E. Farshadfar and F. Nazarian. 2004. Genetic study of some morphophysiological traits in bread wheat lines under dryland conditions using diallel crossing. Seed and Plant Improvement Journal, 20: 429-444 (In Persian).

4. Golabadi, M. and A. Arzani. 2003. Study of Genetic Variation and Factor Analysis of Agronomic Traits in Durum Wheat. Journal of Water and Soil Science, 7: 115-127 (In Persian).

5. Golparyar, A.R., M.R. Ghanadha, A.A. Zali, A. Ahmadi, E.M. Harvan and A. Ghasemi pirbalooti. 2006. Factor analysis of morphological and morpho-physiological traits in bread wheat (Triticum aestivum L.) genotypes under drought and non-drought stress conditions. Journal of Pajouhesh and Sazandegi, 72: $52-59$ (In Persian).

6. Ghulam Mahboob, S. and M.A. Cjowdhry. 2000. Inheritance of Yield and some other MorphoPhysiological Plant Attributes in Bread Wheat irrigated and drought Stress Condition. Pakistan Journal of Biological Sciences, 3: 983-987.

7. Moghaddam, M. and H. Amiri Oghan. 2015. Biometrical Methods in Quantitative Genetic Analysis. 3th edn. Kalyani Publishers, 415 pp.

8. Mostafavi, Kh., R. Choukan, M. Taeb, M.R. Bihamta and E. Majidi Heravan. 2009. Study of the gene action in controlling agronomic traits in maize (Zea Mays L.) -using diallel crossing design. Iranian Journal of Crop Sciences, 10: 331-348 (In Persian).

9. Nabovati, S., M. Aghaee Sarbarzeh, R. Choukan, F. Ghanavati and G. Najafian. 2010. Genetic Variation in Agronomic Characteristics and Grain Quality Traits of Durum Wheat genotypes. Seed and plant Improvement Journal, 26: 331-350 (In Persian).

10. Ojaghi, J. and A. Akhundi. 2010. Genetic Effects for grain yield and its Related Traits in Doubled Haploid Lines of Wheat. International Journal of Agriculture and Biology, 1291: 86-90.

11. Pask, A., A.K. Joshi, Y. Manes, I. Sharma, R. Chatrath, G.P. Singh, V.S. Sohu, G.S. Mavi, V.S.P. Sakuru, I.K. Kalappanavar, V.K. Mishra, B. Arun, M.Y. Mujahid, M. Hussain, N.R. Gautam, N.C.D. Brama, A. Hakim, W. Hoppit, R. Trethowan and M.P. Reynolds. 2014. A wheat phenotyping network to incorporate physiological traits for climate change in South Asia. Field Crops Research, 168: $156-167$.

12. Sadeghi, F. 2014. Estimation of Genetic Structure of Yield and Yield Components in Bread Wheat (Triticum Aestivum L.) using Diallele Method. Journal of Crop Breeding, 6: 101-113 (In Persian).

13. Saeednia, F., M.T. Assad, H. Razi, M. Masumi and E. Ebrahimie. 2012. Study on the inheritance of wheat streak mosaic virus resistance using diallel cross method. Journal of Plant Production, 19: 7390 (In Persian).

14. Sangwan, V.P. and B.D. Chaudhary. 1999. Diallel Analysis in Wheat (T.aestivum). Annals of Biology Ludhiana, 15: 181-183.

15. Tousi Mojarrad, M. and M.R. Ghannadha. 2008. Diallel Analysis for Estimation of Genetic Parameters in Relation to Traits of Wheat Height in Normal and Drought Conditions. Journal of Crop Production and Processing, 12: 143-155 (In Persian).

16. Vanda, M. and S. Houshmand. 2011. Estimation of genetic structure of grain yield and related traits in durum wheat using diallel crossing. Iranian Journal of Crop Sciences. 13: 206-218 (In Persian).

17. Vishwakarma, M.k., V.K. Mishra, P.K. Gupta, P.S. Yadav, H. Kumar and A.K. Joshi. 2014 Introgression of the high grain protein gene Gpc-B1 in an elite wheat variety of Indo-Gangetic Plains through marker assisted backcross breeding. Current Plant Biology, 1: 60-67. 


\title{
Estimation of General and Specific Combining Abilities of Morphological Traits and Grain Yield in Bread Wheat
}

\author{
Bahman Khahani $^{1}$, Mohammad Reza Bihamta ${ }^{2}$ and Behnam Naserian ${ }^{3}$ \\ 1- Graduate M.Sc. Student, Department of Agronomy and Plant Breeding, University of Tehran and Ph.D. Student, \\ Department of Crop Production and Plant Breeding, University of Shiraz \\ 2- Professor, Department of Agronomy and Plant Breeding, University of Tehran \\ (corresponding author: mrghanad@ut.ac.ir) \\ 3- Nuclear Agricultural Research Institute, Department of Plant Breeding, Karaj \\ Receive: March 3, $2016 \quad$ Accepted: November 23, 2016
}

\begin{abstract}
Five wheat varieties were used to estimate gene action for morphological traits and yield and estimate the heritability of traits. The varieties including White Bolani, Brown Bolani, Tajan, Kealk and AS48 mutant have been crossed based on full diallel. The F2 genotypes were planted in a randomized complete block design with three replications in the Nuclear Agricultural Research Institute. Analysis of variance for all the traits revealed that the general combining ability was significant so these traits were controlled by additive genetic effects. The heritability of traits were high and therefore selection based on these will be successful. Additivedominance model was sufficient for this research and non-allelic effect weren't observed, therefore Hayman analysis was performed. Hayman's analysis of variance showed that the additive effects and non-additive effects control the traits. For number of spikelet per spike, second and third internodes diameter, second and third internodes length and leaf area traits, the average degree of dominance indicate that partial dominance controls these traits. Heyman's graphical analysis showed that partial dominance was involved in controlling these traits.
\end{abstract}

Keywords: Additive effect, Diallel analysis, Griffing, Hayman, Wheat 